\section{RESILIENCIA IMPUGNADA: LOS EFECTOS DEL TERREMOTO DE MANABÍ-ECUADOR EN LA PREDISPOSICIÓN A PARTICIPAR EN ESPACIOS DE COOPERACIÓN}

Byron Villacis ${ }^{{ }^{*}}$ y David Vera ${ }^{2}$

\section{RESUMEN}

¿Cuál es el impacto que provocan los desastres sobre la participación en espacios de cooperación? ¿Qué implicaciones tienen estos efectos sobre el entendimiento de la vulnerabilidad social? Al comparar la predisposición a participar en espacios de cooperación antes y después de un terremoto se puede identificar cuán intensa es la hipotética existencia de resiliencia automatizada, entendida como la capacidad que tiene una comunidad para superar circunstancias traumáticas. Manabí, la provincia más poblada del Ecuador, soportó en abril del 2016 un terremoto de $7,8 \mathrm{M}_{\mathrm{w}}$, donde fallecieron más de 600 personas. Utilizando la técnica cuasi-experimental de Diferencias en Diferencias (DiD) se estima el efecto del terremoto en las predisposiciones a formar parte de cooperativas en las poblaciones afectadas por el desastre y luego se las compara con las poblaciones no afectadas. Los resultados permiten concluir que el terremoto no incrementó significativamente esta predisposición; no obstante argumentamos sobre la existencia de factores sociales, económicos y demográficos asociados a la variación. Estos hallazgos permiten proponer que la capacidad de reacción no es automática y depende de factores subyacentes y estructurales. A su vez, los hallazgos permiten contribuir a la interpretación de la conceptualización de la vulnerabilidad social alejándose de nociones individualistas y economicistas.

\section{PALABRAS CLAVES}

Desastres, Vulnerabilidad Social, Impacto terremotos, Cooperativas, Diferencia en Diferencias

\section{CONTESTED RESILIENCE: THE EFFECTS OF THE MANABÍ-ECUADOR EARTHQUAKE IN THE PREDISPOSITION TO PARTICIPATE IN SPACES OF COOPERATION}

\section{ABSTRACT}

What is the impact of disasters on the participation of cooperation spaces? What implications these effects have on the understanding of social vulnerability? By comparing the predisposition to participate in cooperation spaces before and after an earthquake, it is possible to identify how intense is the hypothetical existence of automated resilience -understood as the ability of a community to overcome traumatic circumstances. Manabí, the most populated province in Ecuador, suffered in April 2016 an earthquake of $7.8 \mathrm{M}_{\mathrm{w}}$, where more than 600 people died. Using the quasiexperimental technique of Differences in Differences (DiD), the effect of the earthquake on the predispositions to form part of cooperatives in the populations affected by the disaster is estimated. The results conclude that the earthquake did not increase significantly the predisposition under study. However, there is statistical evidence about social, economic, and demographic factors associated with the variation. The findings support the argument that the capacity of reaction in a social group is not automatic and it depends on underlying and structural factors. In turn, the findings contribute to the interpretation that conceptualizations of social vulnerability, moved away from individualistic and economistic notions, are possible and desirable.

\section{KEYWORDS}

Disasters, Social vulnerability, Earthquake impact, Cooperatives, Difference in Differences
1. Universidad de California - Berkeley, Berkeley, Estados Unidos.

2. Universidad Internacional del Ecuador, Quito, Ecuador.

*Autor de correspondencia: byronvillacis@berkeley.edu

Identificador:

http://revistareder.com/

handle-0719-8477-2020-o8o

\section{RECIBIDO}

30 de octubre de 2020

ACEPTADO

26 de diciembre de 2020

\section{PUBLICADO}

1 de julio de 2021

Formato cita

Recomendada (APA):

Villacis, B. \& Vera, D. (2021). Resiliencia Impugnada:

Los efectos del terremoto de Manabí-Ecuador en la predisposición a participar en espacios de cooperación. Revista de Estudios

Latinoamericanos sobre Reducción del Riesgo de Desastres REDER, 5(2), 125137. http://revistareder.com/ handle-0719-8477-2020-08o

Todos los artículos publicados en REDER siguen una política de Acceso Abierto y se respaldan en una Licencia CreativeCommons Atribución-NoComercial 4.0 Internacional.

Revista de Estudios Latinoamericanos sobre Reducción del Riesgo de Desastres (REDER)

Diseño: Lupe Bezzina 


\section{INTRODUCCIÓN}

¿Qué impacto tienen los desastres en la predisposición de grupos sociales a participar en mecanismos de cooperación? Esta investigación responde a esta pregunta explorando los efectos del terremoto de abril del 2016 en Manabí-Ecuador sobre la predisposición a formar parte de cooperativas o asociaciones. Los resultados de este ejercicio nos permiten contribuir a la discusión sobre las conceptualizaciones de vulnerabilidad social y resiliencia. Tradicionalmente, resiliencia ha sido entendida como la capacidad de reaccionar y recuperarse frente a circunstancias críticas regularmente inesperadas (Briguglio et al., 2009; UNDP, 2011). Contribuciones recientes interpretan a esta capacidad de reacción como un mecanismo inherente de los grupos sociales. Es decir, implícitamente se asume que precondiciones contextuales del grupo afectado no son relevantes; de una u otra forma, la resiliencia aparecerá para adaptarse frente a condiciones críticas o de desastre (Bottrell, 2013; Joseph, 2013). Esta interpretación, implicaría que el rol del Estado queda relegado a un aspecto secundario, y que lo necesario es permitir que los grupos sociales desarrollen estas capacidades de forma autónoma (Rose \& Lentzos, 2017; Welsh, 2014). Este trabajo presenta argumentos para impugnar esta visión y proponer que las capacidades de reacción y cooperación no son inherentes ni automáticas; y por ende, esta predisposición está asociada a factores estructurales inherentes a los grupos sociales afectados por desastres. En esta dirección, el rol del Estado se revela estratégico al momento de preparar y predisponer mecanismos sociales de prevención y protección social, para lo cual se vuelve necesario reivindicar el concepto de vulnerabilidad social en un sentido multidimensional (Gaillard, 2010; Rodríguez Vignoli, 2001).

Para llegar a estas conclusiones ejecutamos un análisis cuantitativo que evalúa el cambio en la predisposición a formar parte de cooperativas y asociaciones antes y después del terremoto en Manabí-Ecuador, país que cuenta con una encuesta oficial a hogares con validez a nivel provincial y de frecuencia anual. Esta encuesta incluye una pregunta que explora la predisposición a formar parte de cooperativas y asociaciones. Estimamos, tanto a nivel descriptivo como inferencial, el cambio en la proporción de esta predisposición antes y después del terremoto, tanto para la provincia de Manabí como para todo el país. Este ejercicio se realizó a través del método de Diferencias en Diferencias (DiD) que permite estimar, por medio de regresiones, el valor de la variación en un sentido causal a través de una lógica semi-experimental. Los resultados demuestran que el terremoto no incrementó significativamente la predisposición a formar parte de cooperativas y asociaciones en Manabí, es decir, no existe evidencia de la existencia de la resiliencia en un sentido mecánico o automático. Estos hallazgos soportan nuestro argumento de que la resiliencia más bien viene dada por factores subyacentes al grupo social estudiado. Esto nos permite desarrollar una discusión sobre el rol del Estado frente a desastres y sobre la conceptualización de vulnerabilidad social.

Lo que resta del documento está dividido en cuatro secciones. En la primera desglosamos el enfoque conceptual en conjunto con una breve revisión de literatura. En la segunda parte planteamos la hipótesis y la metodología del estudio. En la tercera mostramos los resultados y, en la última, describimos una discusión con implicaciones empíricas y teóricas.

\section{REVISIÓN DE LITERATURA Y ENFOQUE CONCEPTUAL}

Esta investigación interpela la noción de resiliencia a través de la reivindicación de la multidimensionalidad del concepto de la vulnerabilidad social. Para ello analiza los efectos de un desastre -en este caso desencadenado por un terremoto- sobre la predisposición a participar en mecanismos de cooperación. En esta sección desarrollamos los conceptos mencionados y las relaciones entre sí, lo cual más adelante nos permite ejecutar el ejercicio empírico que contribuye con elementos para la mencionada impugnación.

El uso moderno del concepto de resiliencia tiene sus orígenes en la literatura de ecología de sistemas, la cual acogió el término de raíces bíblicas que posteriormente fueron utilizadas en la ingeniería mecánica (Alexander, 2013, pp.2708-2710). En ese entonces fue entendida como la capacidad de los sistemas de absorber cambios y perturbaciones manteniendo las relaciones entre las poblaciones (Holling, 1973). La noción fue adaptándose y capturando contribuciones sociológicas (Tobin, 1999) y sicológicas (Garmezy et al., 1984), para dar salto al campo de las estrategias de reducción de riesgos (Lee, 1999). Esta trayectoria tuvo un punto sobresaliente cuando las Naciones Unidas, en la Conferencia Mundial de Reducción de Riesgos de Desastres en Kobe-Japón del 2005, formalizaron una definición en su estrategia para reducción de riesgos 
por desastres: la resiliencia fue definida como la capacidad de un sistema, comunidad o sociedad expuesta a peligros para resistir, absorber, adaptarse y recuperarse de los efectos de un peligro de manera oportuna y eficiente, incluso mediante la preservación y restauración de sus estructuras y funciones básicas (UNISDR, 2009).

La llegada del concepto a campos relacionados con la gobernanza implicó además la discusión sobre la existencia de resiliencia en dominios públicos versus privados. Reportes del gobierno de Estados Unidos (EEUU) sobre sistemas críticos de protección de infraestructura (CIP) en la era de Bill Clinton, empezaron a enfatizar la necesidad de tener alianzas públicoprivadas frente a amenazas y riesgos asociándolas con nociones de vulnerabilidad. Esta visión fue enlazada con la idea de resiliencia de forma explícita en el 2002, después de los ataques del 11 de septiembre del 2001 (Dahlberg, 2015). Para ese entonces, ya el concepto estaba tomando forma como un factor que permite enfrentar desastres, pero cuya presencia implicaba que el grupo social afectado era, aunque sea en parte, el que debía ser el responsable de la reacción (Pelling \& Dill, 2010). No obstante, algunos autores como Gaillard (2010) notaron que se debía prestar más atención a la administración de factores de riesgo que reducen la vulnerabilidad y no solo entender la resiliencia como la capacidad de un sistema de absorber y recuperarse de eventos catastróficos. En este sentido, las víctimas de desastres deben ser incluidas con anticipación en políticas de desarrollo para aprovechar sus capacidades de reconstruir y reducir riesgos por futuros desastres (Gaillard, 2010, p.222).

Es en este espacio donde emerge una discusión entre dos posturas conceptuales: una que aboga por entender a la resiliencia como un mecanismo automático, en el cual una comunidad resiste y se adapta frente a desastres resaltando responsabilidades individuales; lo cual difumina o desvanece -implícita o explícitamente- el rol de sistemas de protección en manos del Estado, regularmente en forma de políticas preventivas (Bottrell, 2013; Joseph, 2013; Rose \& Lentzos, 2017; Welsh, 2014); frente a otra postura que la entiende como parte de un sistema que resiste y se adapta pero abogando por la preservación y restauración de estructuras subyacentes básicas, instituciones y políticas, lo cual implica un rol activo por parte del Estado (Haisch, 2017; Harris et al., 2017; King et al., 2021; Tierney, 2014). Esta investigación se apoya sobre ésta última postura, enfatizando que los mecanismos que reflejan la existencia de resiliencia no aparecen de forma automática, y más bien dependen de estructuras subyacentes que varían de acuerdo a contextos geográficos, temporales y no lineales; así como de capitales económicos, simbólicos y políticos previamente acumulados (Trell, 2018; Uekusa, 2017).

Ahora bien, una vez entendida la postura respecto a la resiliencia, es necesario entender su conexión con la conceptualización de vulnerabilidad social. Nociones contemporáneas la entienden como la combinación de factores que determina el grado en el que la vida o bienestar de un grupo social están en riesgo debido a un evento de la naturaleza o sociedad, lo cual implica la identificación de características del grupo social en términos de su capacidad de anticiparse, enfrentarse, resistir y recuperarse de los impactos mencionados (Busso, 2001; UNDP, 2016; Wisner et al., 2004). Discusiones desde el Sur Global, trazan el origen del concepto para asociarlo a la emergencia de posiciones críticas al concepto de pobreza, la cual regularmente se la entiende como una privación unidimensional de ingresos económicos (Pizarro, 2001). En este sentido, la vulnerabilidad social toma relevancia al momento de convertirse en una identificación multidimensional de condiciones sociales, la cual incluye privaciones o posesión de capitales económicos, políticos, simbólicos y sociales (Gaillard, 2010; Rodríguez Vignoli, 2001). Esta postura no solo permite una caracterización integral de las condiciones de grupos sociales, sino que además visibiliza factores sobre los cuales el Estado ha intervenido previamente, lo que provoca la discusión de su rol activo en la determinación de estructuras socioeconómicas que permiten preservar y restaurar funciones sociales básicas, esenciales al momento de enfrentarse a desastres.

Es sobre este andamiaje conceptual que se soporta esta investigación. Interpelamos la noción de resiliencia para reivindicar la propiedad multidimensional de la vulnerabilidad social, en la cual, la capacidad de resistencia, adaptación y recuperación de una sociedad frente a los efectos de un desastre, dependen de estructuras y funciones esenciales en las cuales el Estado tiene un rol definido. En la siguiente sección enlazamos esta conceptualización con propuestas de operacionalización de la resiliencia, lo que nos permite evaluar su existencia dentro del ejercicio empírico planteado. 


\section{METODOLOGÍA E HIPÓTESIS}

El objetivo de la investigación es impugnar la noción de resiliencia en un sentido automatizado. Para ello, y en el contexto conceptual mencionado en la sección anterior, se requiere evaluar la magnitud del cambio de la resiliencia antes y después de un desastre; lo que requiere una operacionalización del concepto. Cuando se trata de cuantificar esta noción hay propuestas que discuten métricas objetivas (por ejemplo niveles de educación) versus subjetivas (por ejemplo importancia de ritos y tradiciones) (Wilson \& Wilson, 2019), asociaciones con métricas de riesgo y vulnerabilidad (Welle \& Birkmann, 2015), métricas transculturales y en base métodos cuali-cuantitativos (Ungar \& Liebenberg, 2011), dislocación de población en forma de migración (Eriksson et al., 2018), probabilidades reducidas de fallas en terremotos (Bruneau et al., 2003), infraestructura de redes (Reed et al., 2009), métricas genéricas en función del tiempo (Henry \& Ramirez-Marquez, 2012) 0 en función de ambientes de múltiple riesgo (Ayyub, 2015).

Apoyándonos en las propuestas de Bruneau et al. (2003) evaluamos aquí el cambio de resiliencia antes y después de un terremoto, pero utilizando una métrica simplificada que recoge la variación en la predisposición de asociarse o involucrarse en cooperativas. Esta métrica es utilizada como un proxy de la resiliencia debido a que el cooperativismo es entendido como un movimiento que, como su nombre lo indica, busca la cooperación entre sus integrantes a través de la inclusión financiera de los más necesitados (Mogrovejo et al., 2012), ensalzando una doctrina del trabajo en conjunto para lograr objetivos comunes (Larrabure et al., 2011). Consideramos que ésta es una aproximación de la resiliencia puesto que tiene visiones superpuestas al buscar sentidos de comunidad, apoyo mutuo, solidaridad, responsabilidad, libre adhesión e integración (Ratner, 2009; Siapera \& Papadopoulou, 2016). Argumentamos que esta visión está conectada con la resiliencia puesto que ésta última implica un reconocimiento de la existencia de la comunidad y de los mecanismos para resistir, absorber y adaptarse a ambientes de riesgo. Por ende, la predisposición al cooperativismo es un proxy de la resiliencia puesto que faculta la existencia de mecanismos de colaboración y solidaridad con el fin del mantenimiento y reproducción de las funciones sociales básicas del grupo.

Para evaluar la predisposición hacia la participación en cooperativas utilizamos la Encuesta Nacional de Empleo, Desempleo y Subempleo (ENEMDU) del Instituto Nacional de Estadística y Censos del Ecuador (INEC). Esta encuesta se realizaba de forma anual y trimestral en el período 2007-2019. El diseño muestral de la encuesta anual tenía validez provincial, lo que permite realizar comparaciones temporales para la provincia de Manabí con las tendencias del resto de provincias y a nivel nacional. La muestra incluía 31.092 hogares seleccionados bajo muestreo probabilístico en 1.024 centros poblados urbanos y rurales (INEC, 2016). La encuesta incluía la pregunta "¿Usted es socio de alguna cooperativa o asociación?" con las alternativas de respuesta Si y No. Explotamos los resultados de la pregunta y los comparamos en períodos anteriores y posteriores al terremoto de abril del 2016.

Argumentamos que la existencia de resiliencia en un sentido automatizado implicaría que el terremoto generó una capacidad de reacción para recuperarse a través de incrementos en la predisposición a participar en asociaciones disponibles. En nuestro caso, la proporción de personas asociadas a cooperativas en la provincia de Manabí debería incrementarse significativamente después del terremoto, tomando en cuenta un quiebre de tendencia respecto al resto del país. Por lo tanto, la hipótesis de investigación implica la existencia de un incremento en la proporción de respuestas afirmativas a la pregunta de interés al comparar el período previo y posterior al 2016. Para evaluar la hipótesis utilizamos dos caminos: en primer lugar, evaluamos cambios en la proporción de interés incluyendo intervalos de confianza y los comparamos temporalmente (antes y después del 2016) y geográficamente (tendencia de Manabí versus tendencia del resto del país). Posteriormente utilizamos el método de Diferencias en Diferencias (DiD) cuyo diseño cuasiexperimental se utiliza en ciencias sociales para comparar los resultados de un impacto sobre un grupo de control versus un grupo de tratamiento en dos períodos de tiempo (Angrist \& Krueger, 1999). El primer grupo (de control) no esta expuesto a la intervención de interés, en nuestro caso corresponde a las provincias del Ecuador excepto Manabí, a las que llamaremos "Resto País". El segundo grupo (de tratamiento) esta expuesto a la intervención; en nuestro caso será la provincia de Manabí. Los resultados afirmativos a la pregunta de nuestro interés son calculados para identificar la diferencia entre los dos períodos; luego se substrae la diferencia en el grupo de control de la diferencia en el grupo de tratamiento (Glewwe \& Muralidharan, 2016). El supuesto clave del 
procedimiento implica que la ausencia del tratamiento del resultado es solo función aditiva de dos efectos: el efecto del grupo (Manabí versus Resto País) y del efecto temporal (pre terremoto y post terremoto). En nuestro caso:

$$
\begin{aligned}
& Y_{i, s, t}=\text { propensión a cooperatividad en individuo } i \text { en provincia } s \text { en año } t \text { en grupo de } \\
& \text { tratamiento. } \\
& Y_{0, i, s, t}=\text { propensión a cooperatividad en individuo } i \text { en provincia s en año } t \text { en grupo de control. } \\
& \text { donde: }
\end{aligned}
$$

$$
E\left[Y_{0, i s, t} \mid s, t\right]=Y_{s}+\lambda_{t}
$$

bajo el supuesto que no hay diferencias que no estén incorporadas en $Y_{s} \circ \lambda_{t}$ en el modelo:

(2)

$$
Y_{i, s, t}=Y_{s}+\lambda_{t}+\beta D_{i, s, t}+\varepsilon_{i, s, t}
$$

Donde $\beta \mathrm{D}_{i, s, t}$ corresponde a la interacción de ambas diferencias y cuyo coeficiente es el interés del ejercicio. Para ello requerimos dos períodos de tiempo que permiten hacer la comparación, por lo que tomamos el promedio de los tres años previos al terremoto como período inicial $(2013,2014$ y 2015) y el promedio de los años subsiguientes como período posterior (2016, 2017, 2018 y 2019). La ejecución de estos promedios nos permite además evitar problemas de auto correlación serial que usualmente provoca errores estándares artificialmente bajos. Esta técnica es recomendada por Bertrand et al. (2002). En la siguiente sección desglosamos los resultados.

\section{RESULTADOS}

En esta sección desglosamos resultados descriptivos e inferenciales del ejercicio mencionado. Para ello utilizamos el caso del Ecuador que soportó el 16 de abril del 2016 un terremoto de 7,8M $w$ a las $18 \mathrm{~h} 58$ TL (23:58 UTC) en el cantón Pedernales ${ }^{1}$, perteneciente a la provincia de Manabí. Este terremoto fue el más destructivo que haya conocido el país desde 1987. Fallecieron 673 personas, 6.274 resultaron heridas y 28.775 fueron desplazadas. El evento tuvo réplicas con magnitudes de hasta $6,9 \mathrm{M}_{\mathrm{w}}$, registradas a lo largo y ancho de la provincia, afectando incluso las zonas vecinas de Esmeraldas en el norte y Manta-Puerto López en el sur (Instituto Geofísico - EPN, 2018).

De acuerdo a la encuesta ENEMDU, entre el 2013 y 2019, y a su pregunta respecto al cooperativismo y asociación, los cooperativistas o asociados son en su mayoría hombres (72\%), mayores a 36 años (81\%), la mayor parte tienen seguridad social de algún tipo (60\%), tienen más de 10 años trabajando en el mismo sitio (59\%) y sus ingresos son menores a USD\$ 330 al mes (75\%). De acuerdo a la muestra agregada de las ENEMDU del 2013 al 2019, este grupo social representa el 2,3\% de la población mayor a 18 años del país, lo que equivale a alrededor de 260.000 personas. Esta estimación es coherente con registros administrativos de la Superintendencia de Economía Popular y Solidaria que reporta a los asociados y cooperativistas en alrededor de 300.000, al promediar los registros del 2015 y 2019 (SEPS, 2020). Más detalles de esta estructura se pueden observar en la Tabla 1.

Ahora bien, la proporción de interés cambió en el período analizado desde niveles del 4,3\% en el 2013 hasta niveles del 1,6\% en el 2019 a nivel nacional; mientras que en el caso de Manabí pasó, en el mismo periodo, del $3,8 \%$ al 2,0\%. Nuestro objetivo radica en identificar el cambio en la proporción de inscritos en cooperativas o asociaciones antes y después del terremoto del 2016 y diferenciar esta variación entre la provincia de Manabí y el Resto País. Para ello construimos la Tabla 2 que desglosa la proporción de interés en una dimensión temporal previa y posterior al terremoto (2013-2015 versus 2016-2019), y una dimensión territorial para el grupo de control (Resto País) y para el grupo de tratamiento (Manabí).

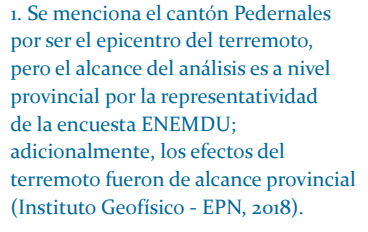




\begin{tabular}{|c|c|c|c|c|}
\hline & & & Cooperati & Asociados \\
\hline & & & $\mathrm{Si}$ & No \\
\hline $\mathrm{n}$ & 422.159 & $100 \%$ & $2,3 \%$ & $97,7 \%$ \\
\hline Sexo & Hombre & $48,3 \%$ & $72,1 \%$ & $58,1 \%$ \\
\hline & Mujer & $51,7 \%$ & $27,9 \%$ & $41,9 \%$ \\
\hline Edad & 18 a 25 & $20,5 \%$ & $3,9 \%$ & $5,9 \%$ \\
\hline & 26 a 35 & $20,6 \%$ & $15,0 \%$ & $17,0 \%$ \\
\hline & 36 a 45 & $19,1 \%$ & $26,0 \%$ & $23,5 \%$ \\
\hline & 46 a 55 & $15,6 \%$ & $26,4 \%$ & $22,4 \%$ \\
\hline & 56 a 65 & $11,5 \%$ & $18,0 \%$ & $17,0 \%$ \\
\hline & Más de 65 & $12,6 \%$ & $10,7 \%$ & $14,2 \%$ \\
\hline Seguridad social & $\mathrm{Si}$ & $36,6 \%$ & $60,6 \%$ & $73,8 \%$ \\
\hline & No & $63,4 \%$ & $39,4 \%$ & $26,2 \%$ \\
\hline Años de trabajo & 0 a 1 & $17,2 \%$ & $7,2 \%$ & $10,7 \%$ \\
\hline & 2 a 5 & $24,7 \%$ & $16,6 \%$ & $20,3 \%$ \\
\hline & 6 a 10 & $17,7 \%$ & $16,9 \%$ & $16,6 \%$ \\
\hline & Más de 10 & $40,4 \%$ & $59,4 \%$ & $52,3 \%$ \\
\hline Ingreso mensual per cápita (US\$) & 0 a 329 & $78,8 \%$ & $75,1 \%$ & $81,9 \%$ \\
\hline & 330 a 1.289 & $19,9 \%$ & $22,0 \%$ & $16,8 \%$ \\
\hline & Más de 1.290 & $1,3 \%$ & $2,9 \%$ & $1,3 \%$ \\
\hline
\end{tabular}

Tabla 1. Estadísticas descriptivas de cooperativistas o asociados para el total de la muestra (2013-2019) Fuente: Autores, 2021, basado en ENEMDU 2013-2019

\begin{tabular}{lccc} 
Cooperativismo & $\begin{array}{c}\text { Pre-terremoto } \\
\mathbf{( 2 0 1 3 - 2 0 1 5 )}\end{array}$ & $\begin{array}{c}\text { Post-terremoto } \\
(\mathbf{2 0 1 6 - 2 0 1 9 )}\end{array}$ & Diferencia \\
\hline Manabí & $2,30 \%$ & $1,50 \%$ & $-0,80 \%$ \\
Resto País & $3,11 \%$ & $1,69 \%$ & $-1,42 \%$ \\
Diferencia & $-0,81 \%$ & $-0,19 \%$ & $0,62 \%$ \\
\hline
\end{tabular}

Tabla 2. Diferencias simples del impacto del terremoto de Manabí en Cooperativismo Fuente: Autores, 2021, basado en ENEMDU 2013-2019

Las columnas de la Tabla 2 muestran los porcentajes promedios de cooperativismo o asociación en los dos períodos de interés (antes y después del terremoto), mientras que las filas muestran los desgloses territoriales analizados (Manabí versus Resto País). Esta comparación nos permite encontrar los dos componentes que integran la estimación inicial de Diferencias en Diferencias (DiD): por una parte, se requiere el cambio en el grupo de tratamiento, resultado de la resta del período anterior y posterior al evento y a ese resultado se le resta la diferencia del cambio en el grupo de control. En nuestro caso, muestran una caída en la proporción de cooperativismo en la provincia de Manabi en $0,80 \%$, mientras que en el Resto País la caída es de 1,42\%. La caída en Manabí se debe en parte al efecto del terremoto; sin embargo, si asumimos que la tendencia del Resto País es lo que hubiera sucedido en Manabí sin terremoto, podemos estimar la DiD como resultado del siguiente procedimiento:

$$
\begin{gathered}
D_{1}=\text { Diferencia por terremoto en Manabí }- \text { Diferencia de la tendencia } \\
D_{2}=\text { Diferencia Resto País Post-terremoto }- \text { Diferencia Resto País Pre-terremoto } \\
\text { DiD }=D_{1}-D_{2}
\end{gathered}
$$

En nuestro caso eso implicaría que la $D_{1}$ es $-0,80 \%$ pero este valor incluye otros efectos, por lo que le sustraemos la diferencia en el resto de país. El resultado es un cambio en la predisposición a ser miembro en cooperativas en Manabí del 0,62\%; asumiendo que la tendencia en el resto del país se aproxima a lo que hubiera pasado en Manabí en la ausencia del terremoto. Según estos 
resultados iniciales, el cooperativismo o asociación se incrementó en Manabí desapegándose de la tendencia a nivel nacional que muestra una caída en el período analizado. Sin embargo, estos resultados pueden ser estimados con mayor precisión utilizando las bases de datos con información individualizada, sobre todo porque nos permite obtener información para saber si el cambio identificado es estadísticamente significativo o no.

Para ello agrupamos las encuestas ENEMDU en dos períodos de tiempo (2013-2015 vs. 20162019), construyendo una base de datos agregada $(n=108.485)$ que incluye tres variables dummy junto con los identificadores de las personas encuestadas y que efectivamente respondieron a la pregunta en cuestión. En el primer caso, la variable $\mathrm{D}^{\text {Tr }}$ asigna valores de 0 cuando el individuo pertenece a un caso de Resto País y 1 cuando pertenece a Manabí. La variable $D^{\text {Post }}$ asigna valores de 0 cuando el individuo corresponde a un caso de las encuestas en el período previo al terremoto y 1 cuando pertenece al período posterior. La última variable es la multiplicación de ambos que permite obtener la interacción. De esta forma podemos ejecutar la regresión cuyo coeficiente $\beta \mathrm{D}_{i, s, t}$ de la ecuación No.2 permitirá identificar el valor del impacto del terremoto en Manabí en la predisposición a ser socio de cooperativas. La Tabla 3 presenta los resultados de la regresión.

\begin{tabular}{|c|c|c|}
\hline & \multicolumn{2}{|c|}{ Variable dependiente: Cooperativismo } \\
\hline & $(01)$ & (2) \\
\hline$\overline{D^{T r}}$ & $\begin{array}{c}-0,007^{* *} \\
(0,003)\end{array}$ & $\begin{array}{c}-0,009^{\star \star *} \\
(0,003)\end{array}$ \\
\hline$D^{\text {Post }}$ & $\begin{array}{c}-0,012^{* * *} \\
(0,001)\end{array}$ & $\begin{array}{c}-0,012^{* * *} \\
(0,001)\end{array}$ \\
\hline ingresopc & & $\begin{array}{c}0,00001^{* * *} \\
(0,00000)\end{array}$ \\
\hline seguro & & $\begin{array}{c}0,013^{* * *} \\
(0,001)\end{array}$ \\
\hline sexo & & $\begin{array}{c}-0,011^{* * *} \\
(0,001)\end{array}$ \\
\hline edad & & $\begin{array}{c}-0,0001^{* * *} \\
(0,00003)\end{array}$ \\
\hline educación & & $\begin{array}{c}0,001^{* *} \\
(0,0003)\end{array}$ \\
\hline tamaño & & $\begin{array}{l}0,001^{* * *} \\
(0,0002)\end{array}$ \\
\hline$D^{\top r}: D^{\text {Post }}$ & $\begin{array}{c}0,004 \\
(0,004)\end{array}$ & $\begin{array}{c}0,004 \\
(0,004)\end{array}$ \\
\hline Constante & $0,029^{\star * \star}$ & $0,040^{* * *}$ \\
\hline & $(0,001)$ & $(0,003)$ \\
\hline Observaciones & 108485 & 108485 \\
\hline $\mathrm{R}^{2}$ & 0,002 & 0,006 \\
\hline $\mathrm{R}^{2}$ Ajustado & 0,002 & 0,006 \\
\hline Error Estándar Residual & $0,148(\mathrm{df}=108481)$ & $0,147(\mathrm{df}=108475)$ \\
\hline Estadístico F & $58571^{* * *}(\mathrm{df}=3 ; 108481)$ & $72919^{\star \star *}(\mathrm{df}=9 ; 108475)$ \\
\hline
\end{tabular}

Nota: ${ }^{*} p<0,1 ;{ }^{* *} p<0,05 ;{ }^{* \star *} p<0,01$

Tabla 3. Modelos de Regresión DiD con y sin variables de control Fuente: Autores, 2021

La regresión que obtiene la DiD -al comparar con el ejercicio analítico descriptivo inicialtiene la ventaja de que arroja los errores estándar de cada estimador, permitiendo interpretar si su diferencia es significativa. En nuestro caso, el coeficiente de interés es $D^{T r}: D^{\text {Post }}=0,004$ con un valor $p$ que comprueba que el cambio no es significativo. Esto quiere decir que -a pesar del terremoto y a pesar de que a nivel descriptivo parecía haber un efecto en Manabí- la regresión permite verificar que no existieron cambios significativos. En otras palabras, podemos rechazar nuestra hipótesis y corroborar que la predisposición a asociarse a cooperativas no responde a una reacción automática en poblaciones como la de Manabí que sufrió el desastre de un terremoto.

Es necesario anotar que el método $\mathrm{DiD}$, a diferencia de otros métodos de análisis causal como el Matching, no supone la inexistencia de factores no observados; por ende, no requiere cumplir supuestos de independencia condicional. Sin embargo, se ejecuta bajo el supuesto clave de que las características no observables son invariantes en el tiempo; es decir, en ausencia del 
tratamiento (en nuestro caso el terremoto), el resultado esperado es sólo una función aditiva de dos efectos: el efecto del grupo (en nuestro caso la provincia) y el efecto del tiempo. Para ello existen, implícitamente, dos supuestos que se aplican en nuestro ejercicio: el primero es que los efectos del terremoto sobre el cooperativismo no tuvieron impacto fuera de Manabí, y que el terremoto tuvo efecto material solamente dentro de Manabí. El segundo supuesto es que las tendencias de cooperativismo, en ausencia del tratamiento, se mantienen con una diferencia constante en las zonas Resto País y Manabí. Esto se puede comprobar de forma empírica solamente para el periodo anterior a la existencia del evento y usualmente se lo realiza de forma visual (Abadie, 2005; Angrist \& Pischke, 2009, pp.169-182). Para ello hemos incluido la Figura 1 que muestra la tendencia de cooperativismo antes y después del evento. La gráfica permite observar que el supuesto se cumple para el período anterior al terremoto y, al parecer, es el evento el que afecta al tratamiento al introducir un cambio en la diferencia. Sin embargo, como se vio en el ejercicio de la regresión, este cambio no resultó significativo; por lo que continuamos con un proceso de inclusión de variables que permiten mejorar el ajuste.

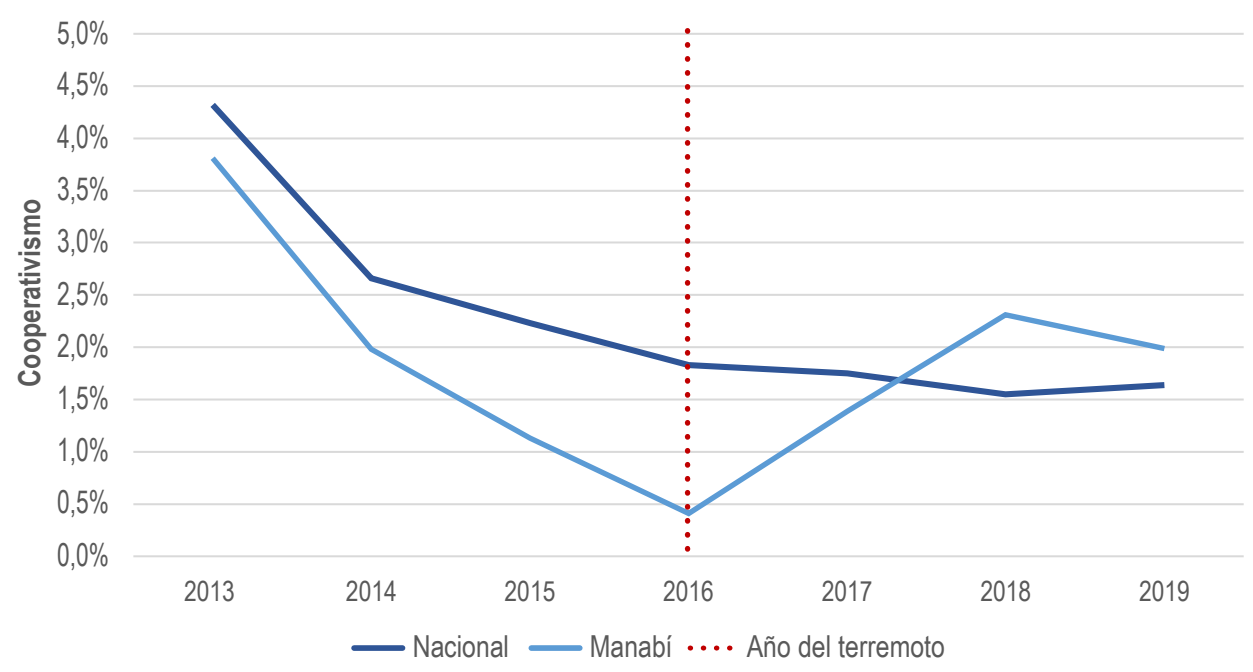

Figura 1. Tendencia de cooperativismo para grupo de interés vs control Fuente: Autores, 2021, basado en ENEMDU 2013-2019

El método de DiD permite agregar variables de control que ayudan a mejorar la estimación del efecto. Para ello agregamos un segundo modelo que incluye variables de control que conceptualmente se asociación a la existencia del cooperativismo con factores subyacentes. Para elegirlas, hay que recordar que la fuente primaria de datos es la encuesta ENEMDU que tiene variables a nivel de personas, vivienda y hogar; sin embargo, la variable de interés "¿Usted es socio de alguna cooperativa o asociación?" se indaga a nivel de persona, por lo que las variables de control las mantenemos al mismo nivel de análisis. Las variables incluidas son:

- Ingreso per cápita.- incluye todos los ingresos mensuales por persona, sea por concepto de trabajos formales o informales, estables o temporales, más cualquier otra fuente de ingreso reportada en la encuesta ${ }^{2}$. Esta reportada en dólares americanos y se espera una relación positiva con la predisposición al cooperativismo y asociación: a mayores ingresos mayor posibilidades de buscar apoyo y apoyar este tipo de esfuerzos colectivos.

- Tenencia de seguro.- incluye a las personas que declaren estar afiliados al seguro ya sea público (IESS, Campesino, de las Fuerzas Armadas, de la Policía) o seguros públicos con y sin hospitalización. Se espera una relación positiva puesto que personas con mayor predisposición al cooperativismo valoran mecanismos de protección social como el de los seguros.

- Sexo.- variable dicotómica que clasifica a los encuestados entre hombres o mujeres. Se espera que exista una relación negativa puesto que, en base a las estadísticas descriptivas, hay mayor propensión al cooperativismo y asociación por parte de los hombres. 
- $\quad$ Edad.- variable continua que registra la edad de los encuestados. En base a estadísticas descriptivas se espera una relación positiva con la predisposición al cooperativismo.

- $\quad$ Nivel de educación.- variable continua que registra el número de años de estudio de los encuestados. Se espera una relación positiva entre las variables debido a que la valoración de beneficios del cooperativismo y asociación van de la mano con el avance en niveles de educación.

- Tamaño de la empresa.- variable continua que registra el número de empleados que tiene la empresa donde actualmente trabaja el encuestado. Se espera una relación positiva puesto que a mayor cantidad de trabajadores mayor posibilidad y necesidad de capitalizar beneficios fruto de proceso de cooperativismo y asociación.

Adicionalmente se incluye estadísticos descriptivos de los contrastes de comparación de medias que evalúan la estabilidad de las covariables en el tiempo; estas se pueden observar de forma proporcional en Cuadro No.1 del Anexo y a través del nivel de significancia de la relación lineal (que permite evaluar el cambio en los períodos de análisis) en Cuadro No.2 del Anexo. Una vez ejecutado el Modelo No.2 podemos observar que el estimador de interés $D^{\text {Tr: }} D^{\text {Post }}$ se mantiene estable tanto a nivel de coeficiente como en el nivel de su valor $p$. Esto que permite concluir con aún mayor seguridad que no existió una variación significativa en la predisposición de cooperatividad y asociación después del terremoto de abril del 2016. Adicionalmente, este modelo incluye variables que permiten especular sobre mecanismos subyacentes que influyen en la dinámica del cooperativismo y asociación. En las variables de control incluidas (ingreso per cápita, seguro, sexo, edad y tamaño del establecimiento) sí existe asociación significativa, en la dirección que se esperaba y al nivel de $p<0.01$. Mientras que en la variable educación también existe relación significativa, pero a un nivel de $p<0.05$. Es decir, condiciones económicas (ingreso per cápita, tamaño de la empresa), demográficas (edad y sexo) y sociales (tenencia de seguro, nivel de educación) ayudan a identificar la estimación de la existencia (o en este caso ausencia) de cambios en el nivel de cooperativismo y asociación debido al terremoto. Sin embargo, se requieren ejercicios posteriores para explorar cuantitativamente los factores que están detrás de la dinámica esta predisposición. Estos resultados se pueden observar en la Tabla 2, donde se observa además una mejora marginal en el ajuste del modelo, medido por el estadístico F, lo cual era de esperarse al agregar más variables con coeficientes significativos. Sin embargo, como se mencionó anteriormente, este modelo busca entender la predisposición de cooperatividad y asociación más que predecirla, y en este sentido las variables de control no modifican significativamente los coeficientes de interés o el error estándar del modelo.
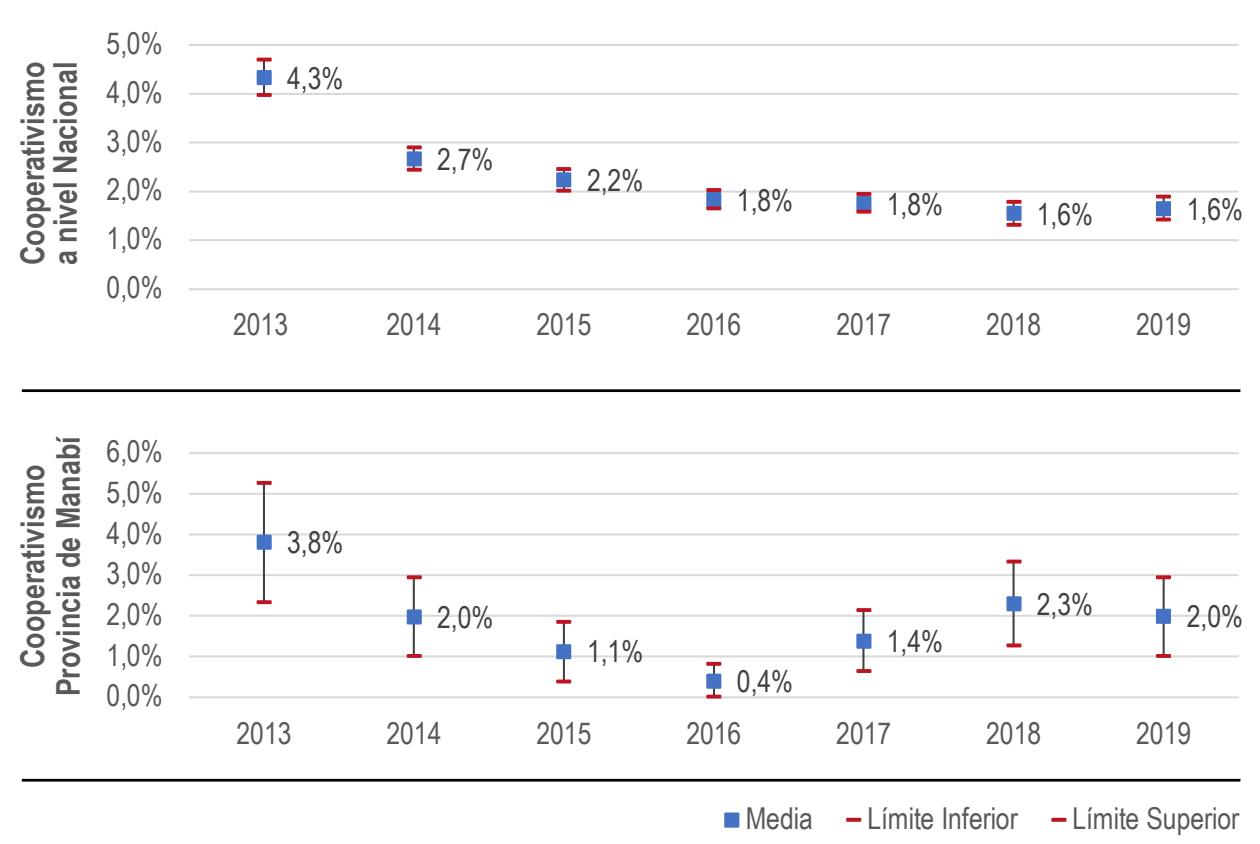
La dinámica de la predisposición al cooperativismo y a la asociación puede observarse en la Figura 2, tanto para el caso Nacional como el de Manabí. Los gráficos, que incluyen intervalos de confianza, ilustran cómo la tendencia decreciente nacional parece diferenciarse en el caso de Manabí justo después de terremoto. Sin embargo, tanto por el análisis de intervalos de confianza del gráfico, como por el análisis de DiD a nivel descriptivo y de regresión, podemos concluir que esta dinámica estuvo influenciada por otros factores subyacentes que no responden a una resiliencia automatizada.

\section{DISCUSIÓN}

La naturaleza ontológica de interpretaciones contemporáneas y neoliberales de resiliencia, entendida como la capacidad de reaccionar y recuperarse frente a circunstancias críticas regularmente inesperadas, recoge mayoritariamente influencias de la psicología (Yates \& Masten, 2004); esto, a pesar de que la genealogía del concepto reconoce una interpretación más amplia y diversa. Esta es una primera pista para entender qué interpretaciones superficiales otorgan mayor valor a reacciones individuales, independientes y autónomas; puesto que la carga de responsabilidad evoca categorías mentales, sensoriales y perceptuales personales. A este primer elemento hay que agregar un factor crítico sobre la temporalidad de su arribo al campo de gobernanza y ciencias sociales. La popularidad del concepto converge con la promoción de ideas que promueven la individualidad, la corresponsabilidad, la austeridad, la templanza y la moderación (Cretney, 2014). Es decir, la resiliencia en interpretaciones modernas emerge como un dispositivo que libera de responsabilidad a factores subyacentes que podrían ser decisivos a la hora de enfrentar circunstancias críticas.

El presente estudio ha propuesto que un desastre no genera resiliencia en un sentido automático; sino que depende de factores subyacentes que facultan la posibilidad de recuperarse. Sin embargo, la interpretación va más allá de un hallazgo cuantitativo. Argumentamos que la esperanza de encontrar efectos de resiliencia automatizada se da debido al despliegue de la aplicación de la resiliencia desde realidades personales hacia complejidades sociales. Cuando enfrentamos problemáticas colectivas, como la de la vulnerabilidad social frente a desastres, nos vemos en la necesidad de re entender los problemas sociales como colectivos y multidimensionales, contrario a visiones individualistas y economicistas. Esta diferenciación tiene al menos tres implicaciones.

En primer lugar, rescata la connotación social de la vulnerabilidad social. A pesar de esta aparente obviedad, visiones economicistas e individualistas despojan el carácter colectivo de realidades sociales. Este fenómeno es una astuta táctica cuyo efecto implica la desmovilización social, la deslegitimación de la participación política y diluye la conciencia de alienación (Harvey, 2014). Si a esto le sumamos las circunstancias del desastre que provoca que se asimilen sucesos negativos como eventos donde no queda más que aceptar y resistir con capacidades individuales 0 , en el mejor de los casos, colectivas pero automatizadas. En caso de que esa capacidad de resistencia no se active, la responsabilidad es del propio grupo y sus individuos, no de las estructuras subyacentes.

Segundo, revaloriza los factores subyacentes que promueven la cohesión social. Si estudiamos científicamente qué es lo que hace a un grupo social reaccionar con sentido de cohesión, visibilizamos herramientas y dispositivos políticos que deben labrarse, y protegerse. Esto facultaría que colectivos puedan protegerse ante riesgos cultivando mecanismos de protección social que serán decisivos en etapas críticas, evitando que la resiliencia sea utilizada como estrategia para transferir costes y contradicciones inherentes del capital hacia las clases explotadas y hacia la naturaleza (Martínez, 2016). Esta es, sin lugar a dudas, una necesidad de subsecuentes aportes a la literatura.

Tercero, evidencia la pertinencia y jurisdicción de la sociología para estudiar la vulnerabilidad social. La comprensión conceptual y empírica de fenómenos sociales movilizadores pasa por objetificar circunstancias que reconozcan su complejidad para insertar herramientas analíticas en contextos históricos, materiales y simbólicos. Negar esta responsabilidad intelectual desemboca en la aceptación de discursos que implícitamente entienden a los sujetos sociales como seres autómatas despojados de realidades culturales y políticas. Esta pertinencia debe además inmiscuirse con metodologías cuantitativas que permitan reconcentrar los debates en las interpretaciones de los hallazgos y no en la elegancia o exclusividad disciplinaria de técnicas. 


\section{REFERENCIAS}

Abadie, A. (2005). Semiparametric Difference-in-Differences Estimators. The Review of Economic Studies, 72(1),1-19.

Alexander, D. (2013). Resilience and disaster risk reduction: an etymological journey. Natural Hazards and Earth System Sciences, 13(11), 2707-2716. http://doi.org/10.5194/nhess-13-2707-2013

Angrist, J.D., \& Krueger, A.B. (1999). Empirical Strategies in Labor Economics. In Handbook of Labor Economics (pp.1277-1366) Vol. 3. Elsevier.

Angrist, J.D., \& Pischke, J.S. (2009). Mostly Harmless Econometrics: An Empiricist's Companion. Princeton University Press.

Ayyub, Bilal. (2015). Practical Resilience Metrics for Planning, Design, and Decision Making. ASCE-ASME Journal of Risk and Uncertainty in Engineering Systems, Part A: Civil Engineering, 1, 04015008. http:// doi.org/10.1061/AJRUA6.0000826

Bertrand, M., Duflo, A., \& Mullainathan, S. (2002). How Much Should We Trust Differences-in-Differences Estimates?. National Bureau of Economic Research.

Bottrell, D. (2013). Responsibilised Resilience? Reworking Neoliberal Social Policy Texts. M/C Journal, 16. http://doi.org/10.5204/mcj.708

Bourdieu, P. (2010). La Producción de La Creencia. Contribución a Una Economía de Los Bienes Simbólicos. En El Sentido Social Del Gusto (pp.153-229). Siglo 21.

Briguglio, L., Cordina, G., Farrugia, N. \& Vella, S. (2009). Economic Vulnerability and Resilience: Concepts and Measurements. Oxford Development Studies, 37(3), 229-47. http://doi. org/10.1080/13600810903089893

Bruneau, M., Chang, S., Eguchi, R., Lee, G., O’Rourke, T., Reinhorn, A., Shinozuka, M., Tierney, K., Wallace, W. \& Winterfeldt, D. (2003). A Framework to Quantitatively Assess and Enhance the Seismic Resilience of Communities. Earthquake Spectra - EARTHQ SPECTRA, 19. http://doi.org/10.1193/1.1623497

Burton, I., Huq, S., Lim, B., Pilifosova, O. \& Schipper, E.L. (2002). From Impacts Assessment to Adaptation Priorities: The Shaping of Adaptation Policy. Climate Policy, 2(2-3),145-59. https://doi.org/10.3763/ cpol.2002.0217

Busso, G. (2001). Vulnerabilidad Social: Nociones e Implicaciones Politicas Para Latinoamérica a Inicios Del Siglo XXI. CELADE-CEPAL.

Cretney, R. (2014). Resilience for Whom? Emerging Critical Geographies of Socio-Ecological Resilience. Geography Compass, 8(9), 627-40. http://doi.org/10.1111/gec3.12154

Dahlberg, R. (2015). Resilience and Complexity: Conjoining the Discourses of Two Contested Concepts. Culture Unbound: Journal of Current Cultural Research, 7(3), 541-57. http://doi.org/10.3384/ cu.2000.1525.1572541

Dow, K. (1992). Exploring Differences in Our Common Future(s): The Meaning of Vulnerability to Global Environmental Change. Geoforum, 23(3), 417-36. http://doi.org/10.1016/0016-7185(92)90052-6

Eriksson, C., Kimber, B. \& Skoog, T. (2018). Design and implementation of RESCUR in Sweden for promoting resilience in children: a study protocol. BMC Public Health, 18, 1250. https://doi.org/10.1186/ s12889-018-6145-7

Gaillard, J. (2010). Vulnerability, capacity and resilience: Perspectives for climate and development policy. $J$. Int. Dev., 22, 218-232. https://doi.org/10.1002/jid.1675

Garmezy, N., Masten, A., \& Tellegen, A. (1984). The Study of Stress and Competence in Children: A Building Block for Developmental Psychopathology. Child Development, 55(1), 97-111. https://doi. org/10.2307/1129837

Glewwe, P., \& Muralidharan, K. (2016). Improving Education Outcomes in Developing Countries. In Handbook of the Economics of Education (pp.653-743) Vol. 5. Elsevier.

Haisch, T. (2017). Interplay between ecological and economic resilience and sustainability and the role of institutions: evidence from two resource-based communities in the Swiss Alps. Resilience, 6(3). 1-15. http://doi.org/10.1080/21693293.2017.1417679

Harris, L. Eric K. Chu \& Ziervogel, G. (2018). Negotiated resilience. Resilience, 6(3). 196-214. http://doi. org/10.1080/21693293.2017.1353196

Harvey, D. (2014). Diecisiete Contradicciones y El Fin del Capitalismo. IAEN Quito. 
Henry, D. \& Ramirez-Marquez, J. (2012). Generic Metrics and Quantitative Approaches for System Resilience as a Function of Time. Reliability Engineering E System Safety, 99, 114-122. http://doi. org/10.1016/j.ress.2011.09.002

Holling, C.S. (1973). Resilience and Stability of Ecological Systems. Annual Review of Ecology and Systematics, 4(1), 1-23. https://doi.org/10.1146/annurev.es.04.110173.000245

INEC. (2016). Encuesta Nacional de Empleo, Desempleo y Subempleo - ENEMDU - Manual Metodológico. INEC.

Instituto Geofísico - EPN. (2018). Dos años después del Terremoto de Pedernales: actualización sísmica - Instituto Geofísico - EPN. Recuperado de: https://www.igepn.edu.ec/interactuamos-conusted/1572-dos-anos-despues-del-terremoto-de-pedernales-actualizacion-sismica

Joseph, J. (2013). Resilience as embedded neoliberalism: a governmentality approach. Resilience, 1(1), 38-52. http://doi.org/10.108o/21693293.2013.765741

Kaztman, R., Beccaria, L., Filgueira, F., Golbert, L., Kessler, G., ILO Multidisciplinary Technical Advisory Team, \& Ford Foundation. (1999). Vulnerabilidad, activos y exclusión social en Argentina y Uruguay. Equipo Técnico Multidisciplinario para Argentina, Brasil, Chile, Paraguay y Uruguay.

King, H., Crossley, S., \& Smith, R. (2021). Responsibility, resilience and symbolic power. The Sociological Review. https://doi.org/10.1177/0038026120986108

Larrabure, M., Vieta, M. \& Schugurensky, D. (2011). The 'New Cooperativism' in Latin America: WorkerRecuperated Enterprises and Socialist Production Units. Studies in the Education of Adults, 43(2), 181-196. http://doi.org/10.1080/02660830.2011.11661612

Lee, K.N. (1999). Appraising adaptive management. Conservation Ecology, 3(2), 3. https://doi. org/10.5751/ES-00131-030203

Lentzos, F., \& Rose, N.S. (2017). Making us resilient: Responsible citizens for uncertain times. In S. Trnka, \& C. Trundle (Eds.), Competing Responsibilities: The Ethics and Politics of Contemporary Life. Duke University Press.

Martínez, J.A.F. (2016). La Trampa Neoliberal de La Resiliencia. Papeles de Relaciones Ecosociales y Cambio Global, (134), 129-38.

Meerow, S., Newell, J.P., \& Stults, M. (2016). Defining Urban Resilience: A Review. Landscape and Urban Planning, 147, 38-49. http://doi.org/10.1016/j.landurbplan.2015.11.011

Mogrovejo, R., Mora, A. \& Vanhuynegem, P. (2012). El cooperativismo en América Latina. Una diversidad de contribuciones al desarrollo sostenible. Oficina de la OIT para los Países Andinos.

Oxford Development Studies. (2009). Vulnerability in Development. Advances in Concept and Measurement, 37(3).

Pelling, M. \& Dill, K. (2010). Disaster Politics: Tipping Points for Change in the Adaptation of Sociopolitical Regimes. Progress in Human Geography, 34(1), 21-37. http://doi. org/10.1177/0309132509105004

Pizarro, R. (2001). La vulnerabilidad social y sus desafíos: una mirada desde América Latina. CEPAL División de Estadística y Proyecciones Económicas.

Ranci, C. (2010). Social Vulnerability in Europe: The New Configuration of Social Risks. Palgrave Macmillan.

Ratner, C. (2009). Cooperativism: A Social, Economic, and Political Alternative to Capitalism. Capitalism Nature Socialism, 20, 44-73.

Reed, D., Kapur, K. \& Christie, R. (2009). Methodology for assessing the resilience of networked infrastructure. IEEE Systems Journal, 3(2), 174-180. http://doi.org/10.1109/JSYST.2009.2017396

Rodríguez, C.A.R., Labrador Machín, O. \& Alemán, J.L.A. (20o6). El Cooperativismo. Revista Idelcoop, 33(168).

Rodríguez Vignoli, J. (2001). Vulnerabilidad y grupos vulnerables: un marco de referencia conceptual mirando a los jóvenes. Naciones Unidas, CEPAL, Proyecto Regional de Población CELADE-FNUAP, Centro Latinoamericano \& Caribeño de Demografía (CELADE), Div. de Población.

Schmidtlein, M.C., Deutsch, R.C., Piegorsch, W.W. \& Cutter, S.L. (2008). A Sensitivity Analysis of the Social Vulnerability Index. Risk Analysis, 28(4), 1099-1114. http://doi. org/10.1111/j.1539-6924.2008.01072.X 
SEPS. (2020). Actualidad y Cifras Economia Popular y Solidaria. SEPS.

Siapera, E. \& Papadopoulou, L. (2016). Entrepreneurialism or Cooperativism?. Journalism Practice, 10(2), 178-195. http://doi.org/10.1080/17512786.2015.112576o

Tate, E. (2013). Uncertainty Analysis for a Social Vulnerability Index. Annals of the Association of American Geographers, 103(3), 526-43. http://doi.org/10.1080/00045608.2012.700616

Tierney, K. (2014). The Social Roots of Risk: Producing Disasters, Promoting Resilience. Stanford University Press.

Tobin, G. (1999). Sustainability and community resilience: the holy grail of hazards planning?. Global Environmental Change Part B: Environmental Hazards, 1(1), 13-25. https://doi.org/10.3763/ ehaz.1999.0103

Trell, E-M., Restemeyer, B., Bakema, M. \& van Hoven, B. (2018). Governing for resilience in vulnerable places - an introduction. Routledge. https://doi.org/10.4324/9781315103761

Uekusa, Shinya. (2018). Rethinking resilience: Bourdieu's contribution to disaster research. Resilience, 6(3), 181-195. http://doi.org/10.1080/21693293.2017.1308635

Ungar, M. \& Liebenberg, L. (2011). Assessing Resilience Across Cultures Using Mixed Methods: Construction of the Child and Youth Resilience Measure. Journal of Mixed Methods Research, 5(2), 126-149. http://doi.org/10.1177/1558689811400607

United Nations and Development Programme - UNDP. (2011). Towards Human Resilience: Sustaining MDG Progress in an Age of Economic Uncertainty. United Nations Publications.

UNPD. (2016). Putting People First: Practice, Challenges and Innovation in Characterizing and Mapping Social Groups. UNDP.

Welle, T. \& Birkmann, J. (2015). The World Risk Index. JoEE, 2(1), 1550003. http://doi.org/10.1142/ S2345737615500037

Welsh, M. (2014). Resilience and responsibility: Governing uncertainty in a complex world. The Geographical Journal, 18o(1), 15-26. http://doi.org/10.1111/geoj.12012

Wilson, G. \& Wilson, O. (2019) Assessing the resilience of human systems: a critical evaluation of universal and contextual resilience variables. Resilience, 7(2), 126-148. http://doi.org/10.1080/216932 93.2018.1539205

Wisner, B., Blaikie, P., Cannon, T., \& Davis, I. (2004). At Risk: Natural Hazards, People’s Vulnerability, and Disasters (2nd ed). Routledge.

Yates, T.M., \& Masten, A.S. (2004). Fostering the Future: Resilience Theory and the Practice of Positive Psychology. In Positive psychology in practice (pp.521-39). John Wiley \& Sons Inc. 